\title{
Anomalous Diamagnetism of $\mathrm{YbPb}_{3}$ Compound: Pressure Effects
}

\author{
A.E. Baranovskiy ${ }^{a}$, G.E. Grechnev ${ }^{a}$, A.S. Panfilov ${ }^{a}$, \\ I.V. SvechKarev ${ }^{a}$, O. Żogą ${ }^{a}$, A. CzOpniK ${ }^{b}$ And A. \\ HACKEMER $^{b}$
}

${ }^{a}$ B. Verkin Institute for Low Temperature Physics and Engineering 61103 Kharkov, Ukraine

${ }^{b}$ W. Trzebiatowski Institute of Low Temperature and Structural Research 50-950 Wrocław, Poland

The effect of a uniform pressure on the magnetic susceptibility was measured for $\mathrm{YbPb}_{3}$ compound, wherein a degeneracy point of the energy bands is located just below the Fermi level and responsible for the anomalous diamagnetism. Theoretical analysis of the experimental data has revealed that a pronounced increase of diamagnetism with pressure is governed by closing the degeneracy point towards the Fermi energy

PACS numbers: 75.10.Lp, 75.80.+q

\section{Introduction}

The detailed calculations of the band structure in isostructural and isovalent $\mathrm{AuCu}_{3}$-type compounds $\mathrm{CaSn}_{3}, \mathrm{CaPb}_{3}, \mathrm{YbSn}_{3}$, and $\mathrm{YbPb}_{3}$ (where Yb atoms are divalent) $[1,2]$ revealed a degeneracy point of the energy bands $E_{\mathrm{d}}$ in the vicinity of
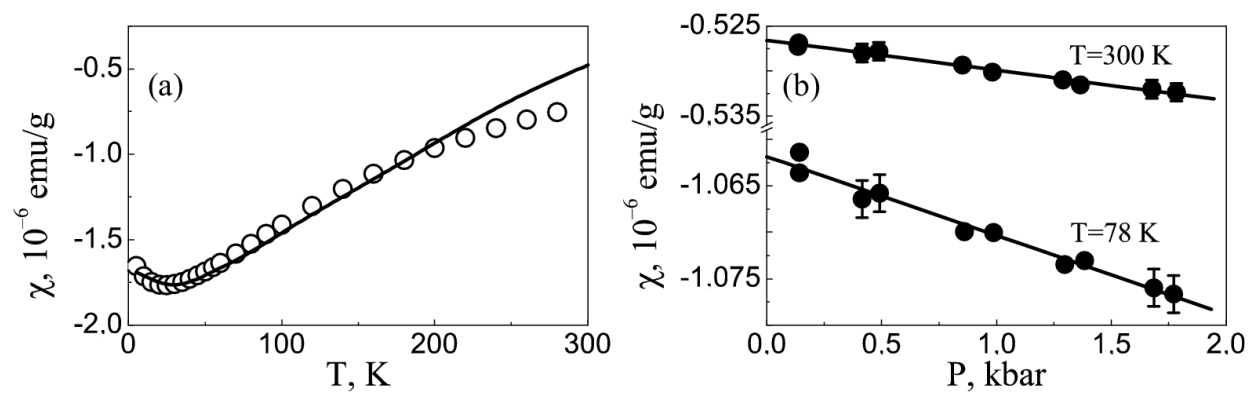

Fig. 1. Experimental (o) and theoretical (solid line) magnetic susceptibility of $\mathrm{YbPb}_{3}$ [1] (a) and its pressure dependence at 78 and $300 \mathrm{~K}$ (b). 
the Fermi level $E_{\mathrm{F}}$. According to the existing theory of the orbital susceptibility for degenerated bands $[3,4]$ this peculiarity gives rise to an anomalous diamagnetism with a distinctive temperature dependence, which was observed experimentally in these systems (see e.g. [1, 5]). The real magnitude of the effect is strongly governed by the parameter $\xi=E_{\mathrm{F}}-E_{\mathrm{d}}$, being a giant at $E_{\mathrm{F}}=E_{\mathrm{d}}, T=0 \mathrm{~K}$. In $\mathrm{YbPb}_{3}$ compound $E_{\mathrm{F}}$ is so close to $E_{\mathrm{d}}$ that the anomalous diamagnetism dominates in its magnetic susceptibility [1, 2] (see Fig. 1a). Here we report results of a study of the pressure effect on magnetic susceptibility and electronic structure of $\mathrm{YbPb}_{3}$ to gain a better insight into the nature of the anomalous magnetism in this and similar $R M_{3}$ compounds.

\section{Results and discussion}

The $\mathrm{YbPb}_{3}$ sample was grown by a similar to [6] method in the form of druse involving a few single crystals of $1-3 \mathrm{~mm}$ in size immersed in an excess of $\mathrm{Pb}$ with total mass of about $0.7 \mathrm{~g}$. The measurements of susceptibility $\chi$ were carried out under helium gas pressure up to $2 \mathrm{kbar}$ at fixed temperatures 78 and $300 \mathrm{~K}$ by using the pendulum-type magnetometer placed into a non-magnetic pressure cell [7]. The relative errors did not exceed $0.2 \%$. Experimental dependences $\chi(P)$ (Fig. 1b) show a linear increase of diamagnetism with pressure. The corresponding pressure derivatives of susceptibility are listed in Table.

\section{TABLE}

Magnetic characteristics of $\mathrm{YbPb}_{3}$ compound: $\chi_{0}$ is intrinsic susceptibility according to work $[1], \chi_{\text {meas }}$ and $\mathrm{d} \chi_{\text {meas }} / \mathrm{d} P$ measured values of the sample susceptibility and its pressure derivative, $\mathrm{d} \chi_{0} / \mathrm{d} P$ pressure derivative of the intrinsic susceptibility, and $\mathrm{d} \chi_{\text {theor }} / \mathrm{d} P$ calculated pressure effect.

\begin{tabular}{c|c|c|c|c|c}
\hline \hline$T$ & $\chi_{0}$ & $\chi_{\text {meas }}$ & $\mathrm{d} \chi_{\text {meas }} / \mathrm{d} P$ & $\mathrm{~d} \chi_{0} / \mathrm{d} P$ & $\mathrm{~d} \chi_{\text {theor }} / \mathrm{d} P$ \\
\hline $\mathrm{K}$ & \multicolumn{2}{|c|}{$10^{-6} \mathrm{emu} / \mathrm{g}$} & \multicolumn{3}{|c}{$10^{-6}(\mathrm{emu} / \mathrm{g}) / \mathrm{Mbar}$} \\
\hline 78 & -1.542 & -1.062 & $-8.5 \pm 1.5$ & $-11.1 \pm 2$ & -13.3 \\
\hline 300 & -0.715 & -0.526 & $-3.4 \pm 1$ & $-4.4 \pm 1.5$ & -3.3
\end{tabular}

It should be noted that our values of the magnetic susceptibility at ambient pressure somewhat differ from the data of [1] for the $\mathrm{YbPb}_{3}$ single crystal of high quality (see Table). That is obviously due to the presence in our sample, of the free $\mathrm{Pb}$ foreign phase and a small amount of $\mathrm{Yb}_{2} \mathrm{O}_{3}$ impurity, which contribute the temperature independent weak diamagnetism of $\mathrm{Pb}$ and the Curie-Weiss paramagnetism [5] of $\mathrm{Yb}^{3+}$ ions. From analysis of the difference in $\chi$ at two temperatures from Table the weight contents of $\mathrm{Pb}$ and $\mathrm{Yb}_{2} \mathrm{O}_{3}$ were estimated to be about $23 \%$ and $1 \%$, respectively. Despite the relatively large content of the free $\mathrm{Pb}$ in the sample, its contribution to the measured pressure effect is negligible because of 
a mainly ionic nature of $\mathrm{Pb}$ diamagnetism. As to the paramagnetism of $\mathrm{Yb}_{2} \mathrm{O}_{3}$, its pressure dependence, arisen from the volume effect on the paramagnetic Curie temperature $\theta(\approx-3 \mathrm{~K}[5])$, appears to be well below the experimental error bars. Hence the evaluations of the pressure derivative for the intrinsic susceptibility of $\mathrm{YbPb}_{3}$ from the measured effect (Table) include only corrections for the weight content of the $\mathrm{YbPb}_{3}$ compound in the sample.

As it has been established earlier $[1,2]$, the dominant contribution to the magnetic susceptibility $\chi\left(\equiv \chi_{0}\right)$ of $\mathrm{YbPb}_{3}$ is the orbital diamagnetism $\chi_{\mathrm{d}}$ of degenerated bands. Within two-band approximation of the spectrum in the vicinity of the critical point $E_{\mathrm{d}}$ (Fig. $\left.2 \mathrm{a}\right) \chi_{\mathrm{d}}$ can be calculated by [2]

$$
\chi_{\mathrm{d}}=A \int_{0}^{\xi_{0}} \frac{\mathrm{d} \varepsilon}{\varepsilon}\left[\frac{1}{1+\exp \left(\frac{-\varepsilon-\xi}{T}\right)}-\frac{1}{1+\exp \left(\frac{\varepsilon-\xi}{T}\right)}\right] .
$$

Here $A$ is ab initio calculated scaling coefficient, $\xi_{0}$ - so-called cutoff parameter, and $\xi=E_{\mathrm{F}}-E_{\mathrm{d}}$. By including in (1) the effect of electron scattering on defects of the lattice in terms of the effective temperature $T_{\mathrm{sc}}$ added to the real $T$, the best fit of (1) to the experimental $\chi(T)$ data [1] has been obtained with $\xi \simeq 0.7 \mathrm{mRy}$ and $T_{\mathrm{sc}} \simeq 50 \mathrm{~K}[2]$. Since the $\chi_{\mathrm{d}}$ magnitude is mainly determined by the $\xi$ value, the pressure effect on $\chi$ is assumed to be governed by the pressure dependence of $\xi(P)$ and can be described as

$$
\frac{\mathrm{d} \chi}{\mathrm{d} P} \simeq \frac{\mathrm{d} \chi_{\mathrm{d}}}{\mathrm{d} P} \simeq \frac{\partial \chi_{\mathrm{d}}}{\partial \xi} \frac{\mathrm{d} \xi}{\mathrm{d} P}
$$
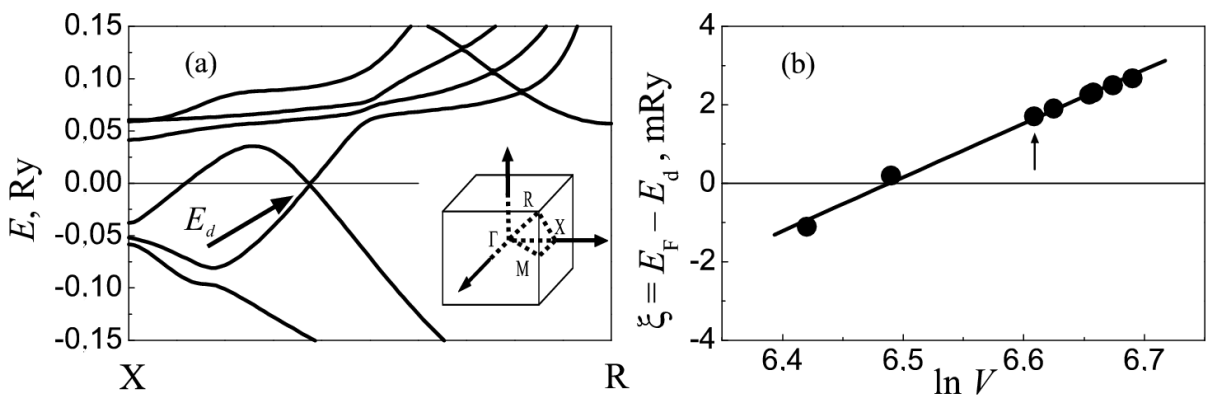

Fig. 2. (a): Calculated band structure of $\mathrm{YbPb}_{3}$ along the XR direction of the Brillouin zone (see insert) in the vicinity of the Fermi level $E_{\mathrm{F}}=0$ [2]. (b) Volume dependence of the relative position of $E_{\mathrm{F}}$ and degeneracy point $E_{\mathrm{d}}$. The data for the equilibrium unit-cell volume at ambient pressure are marked by arrow.

The ab initio full potential linear muffin-tin orbital (FP-LMTO) band structure calculations for $\mathrm{YbPb}_{3}$ (see[2] for details) are carried out for a number of the lattice parameters close to the experimental value. This has allowed us to determine the volume dependence of $\xi$ (Fig. 2b) and the corresponding pressure derivative $\mathrm{d} \xi / \mathrm{d} P \simeq-23 \mathrm{mRy} / \mathrm{Mbar}$, by using the calculated value of the bulk 
modulus $B=0.53$ Mbar. By substitution of this $\mathrm{d} \xi / \mathrm{d} P$ value into (2), together with the value of $\partial \chi_{\mathrm{d}} / \partial \xi$ evaluated within (1) with the above-mentioned set of $\xi$ and $T_{\mathrm{sc}}$ data, the obtained estimates for the pressure effect of $\mathrm{d} \chi / \mathrm{d} P$ appeared to be in good agreement with the experimental results (see Table).

\section{Summary}

In summary, the experimental and theoretical studies of the pressure effect on $\chi$ in $\mathrm{YbPb}_{3}$ have revealed that the pronounced increase of the anomalous diamagnetism with pressure is caused by decreasing the energy separation between $E_{\mathrm{F}}$ and the degeneracy point $E_{\mathrm{d}}$. The initial $E_{\mathrm{F}}$ position has been unambiguously determined to be above $E_{\mathrm{d}}$ energy. The obtained estimate of $\mathrm{d} \xi / \mathrm{d} P \simeq-23 \mathrm{mRy} / \mathrm{Mbar}$ together with the best fit initial value of $\xi \simeq 0.7 \mathrm{mRy}$ from [2] allow to expect a topological electronic phase transition in $\mathrm{YbPb}_{3}$ at $P \approx 30 \mathrm{kbar}$, which is accompanied by closing the small electron "pockets" at the XR line of the Brillouin zone and a peak of the anomalous diamagnetism.

\section{References}

[1] A.E. Baranovskiy, G.E. Grechnev, I.V. Svechkarev, A. Czopnik, Czech. J. Phys. 54, D355 (2004).

[2] A.E. Baranovskiy, G.E. Grechnev, I.V. Svechkarev, Low Temp. Phys. 32, 849 (2006).

[3] G.P. Mikitik, I.V. Svechkarev, Sov. J. Low Temp. Phys. 15, 165 (1987).

[4] G.P. Mikitik, Yu.V. Sharlai, Low Temp. Phys. 26, 39 (2000).

[5] J.P.C. Klaasse, F.R. de Boer, P.F. de Châtel, Physica B 106, 178 (1981).

[6] V.B. Pluzhnikov, G.E. Grechnev, A. Czopnik, O. Eriksson, LowTemp. Phys. 31, 313 (2005).

[7] A.S. Panfilov, I.V. Svechkarev, L.F. Romasheva, Low Temp. Phys. 19, 200 (1993). 